\title{
A design of pulse condition simulation device based on squeeze mode
}

\author{
H.D. LIU \& D.S. YAN \& Q.F. DENG \& J.S. LIN \& W.R. SUN
}

School of Electrical and Information Engineering Jinan University, 206 Qianshan Rd. Zhuhai, Guangdong, China

KEYWORD: TCM Pulse-taking; Pulse simulation; STM32; PID control

ABSTRACT: With a view to the fact that the traditional Chinese medicine (TCM) pulse diagnosis learning is difficult, which takes a lot of time as well, and the current pulse simulation device is expensive, it is hard to popularize the device to help the TCM teaching and learning. In this paper, bionics, mechanics, hydraulic systematic, computer technology and sensor technology will be integrated to design a new pulse simulation device with low cost and high precision, which successfully simulates several types of human pulse. In the meantime, an auxiliary developed software will be introduced in the teaching, learning and examination of pulse diagnosis. The project has got great achievement that it has high value of practical application and great prospects, and will help to advance the development of education and research of TCM as well.

\section{Introduction}

In normal TCM pulse-taking teaching, the essential features of the human pulse patterns are described in detail. However, the course provides students with little practical experience, which has been a defect in the succession of traditional Chinese medicine. So developing pulse condition simulators has become a hot issue among TCM researchers. There are two essential types of pulse condition simulation system in China and abroad[1-6]. One is to simulate the effect of pulse condition using hydraulic pressure system, and the other is to simulate it using the vibrations produced by mechanical structure. While those systems gradually improved the precision of the simulation to human pulse after considering fully of its device features, the intelligence of the pulse condition simulation still need to be improved and the cost still need to be reduce. This paper introduce a new design of pulse condition simulation instrument used in teaching with low cost and high precision after doing market survey roundly. It combines all these merits but does not have the defects. It will benefit to shorten time of learning feeling pulse, reduce the learning curve of TCM and increase efficiency of studying TCM and the skills of feeling pulse. It will also be conductive to the development of sphygmology and scientific investigation.

\section{The design of the system}

This is a design of low-power and intelligentized pulse simulation (shown in Figure 1), established on the basis of physiology and Chinese medicine, fused with the technology of modern medical signal detection, computer, and the subject of flushbonading, mechanical, hydromechanics. The simulation platform of heart and meridian blood circulation system are designed to use micro controller unit(MCU) to imitate cardiac automaticity, use the bladder with liquid(the bladder will be named artificial blood chamber) with the help of mechanical structure and pump to imitate heart, and use check valve to imitate the membrane flap of heart and stop the liquid to flow backwards. Moreover, a small size of latex tube is used to imitate blood vessel. When the system works, the pump provides the power of flowing back, and the power plant squeeze artificial blood chamber, producing a variable hydraulic pressure as a result, and the hydraulic pressure flow through the hydraulic system and pour into imitate blood vessel, making the artificial blood vessel filling or shriveled, consequently, producing the simulative pulse. Furthermore, to make the education of pulse-taking more visualized and intelligentized, a visible and manageable upper-computer software, which is based on LabVIEW, is written to show pulse waveform and realize the function of test on-line. 


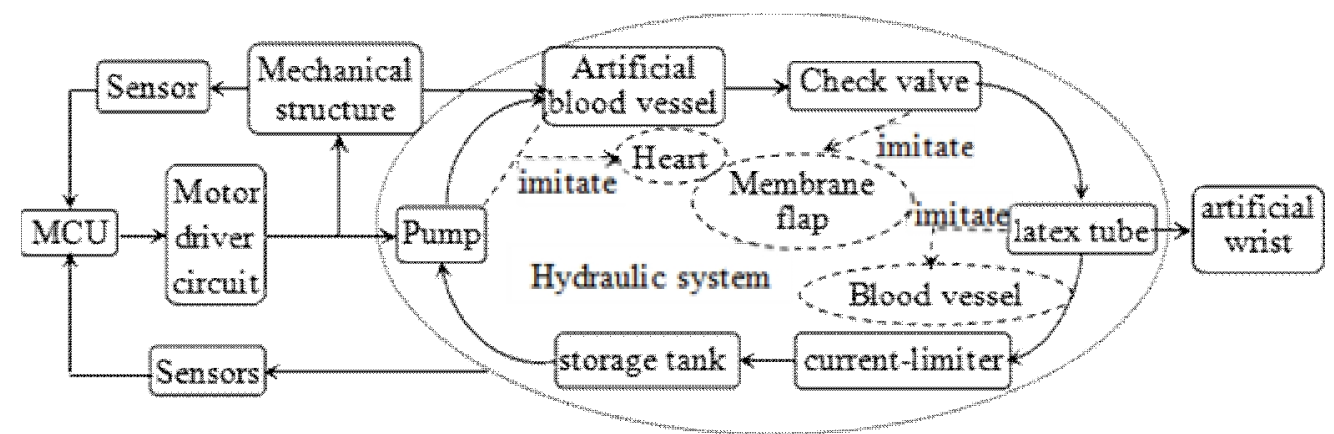

Figure 1. The schematic diagram of pulse imitation device

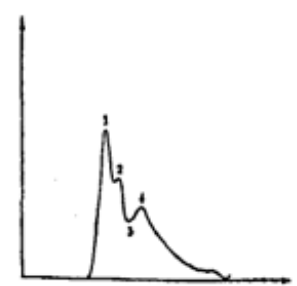

Figure 2. Figure of pulse condition[6]

The simulation of pulse feature: Figure 2 shows a kind of basic pulse waveform. When the heart contracts, the curve rises abruptly, and then the curve declines slowly with one or two small peaks. Implementation method: The MCU controls the splint to move fast and push the artificial blood chamber, which causes a sudden change in the liquid pressure. And then the pressure signal is transmitted to artificial blood vessel through rubber pipe. After that, the splint goes back slowly to reduce the pressure of the inner artificial blood chamber. At the same time, the water pump works to make the water flow back to the artificial blood chamber. And, in order to simulate the small peaks, the MCU has to coordinate the speed of the splint and the frequency of the pump. The program runs periodically to make the system simulate the manifestation of human's pulse.

The simulation of different kinds of pulse: Above all, the design of this control scheme is largely related to the mechanical structure. In order to simulate different intensities of pulse, the program controls the squeeze velocity of the splint to achieve different effects of extrusion. The reflux flow is controlled by the pumping speed of the pump and the time it works. The shunt volume is determined by the time that the magnetic valve works. By controlling the working time of all parts of the system, it can simulate different frequency of pulse. Moreover, the depth of pulse can be realized by adjusting the position of the artificial vessel.

\section{The design of the hardware}

The hardware of the system is composed of four modules, including mechanical transmission module, control circuit module, pint-sized hydraulic system module and artificial hand model. The internal structure of the system is shown in Figure 3.

\section{Mechanical structure}

Mechanical transmission structure is assembled with DC motor with large torque and high rotation speed and screw-sliding table through a couplings[7]. Splint is installed on the sliding table. In order to reduce the influence of friction force between sliding table and screw rod, sliding table and pedestal are fixed with linear bearing. Regular counterclockwise and clockwise movement of the motor with a given speed drives the sliding table to squeeze artificial blood chamber, which realistically reappear the systolic and diastolic of human heart. The mechanical part is made by silicon steel and aluminium alloys which are so stable and reliable that can support high pressure. 


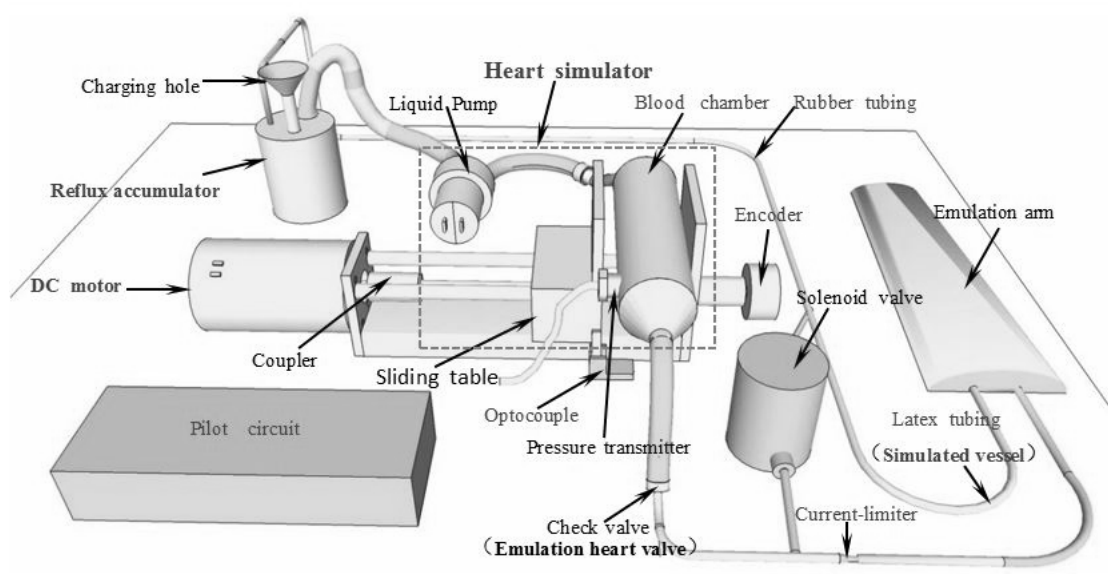

Figure 3. System hardware structure

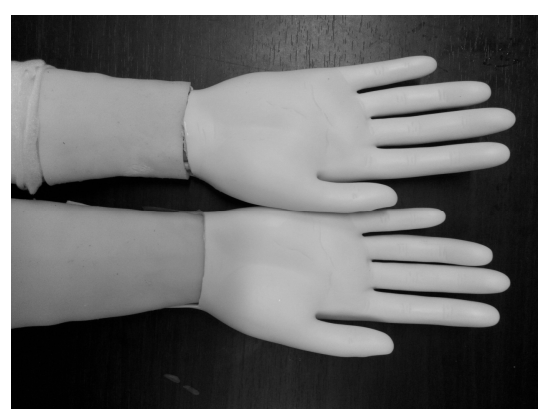

Figure 4. Artificial hand

\section{Pint-sized hydraulic system}

Hydraulic system, including the micro pump, the artificial blood chamber, magnetic valve, liquid storage tank and rubber hoses which connect all parts of the hydraulic system, is a key part for simulating the system of heart and meridian blood circulation system. Specially, the artificial blood vessels inside the artificial hand is made of soft and small latex tube [1,3,5]. All parts of the hydraulic system is connected into a ring. The check valve is adopted to simulate the human heart periosteal flap, so the liquid can only flow in one direction. What's more, the flow rate of liquid is under control by the flow limited component, which can realistically simulate the heart vessel structure and blood flow model. All above condition has laid the foundation for simulating human pulse with high precision.

\section{Hardware circuit}

On the basis of the function of the overall design of system, hardware circuit mainly includes the main controller, motor driver module, sensors module, input control module and power module.

The STM32F401 of ST Semiconductor company is adopted as the center controller. Power module is supplied by $24 \mathrm{~V}$ DC voltage regulator module, and LM2576 chip is selected to design into buck circuit converting $24 \mathrm{~V}$ to $12 \mathrm{~V}$ and $5 \mathrm{~V}$. It only needs few peripheral devices to built efficient regulating circuit, and three different value of voltage are set for the need of different equipment. Motor driver module includes 24V DC motor driver circuit[8] (to make the motor work), and $12 \mathrm{~V}$ driver circuit[9] (to make pump and magnetic valve work). In consideration of characteristic of peripheral devices such as power, VNH3SP30 chip is selected to design into 24V driver circuit and L298N chip is selected to design into 12V driver circuit. Additionally, a variety of protective measures are taken to guarantee the reliability and security of the circuit.

Data acquisition and interaction modules include the sensors and the human-computer interaction interface. The STM32 reads the value of voltage from its pins which connect to the sensors. The human-computer interaction interface comprises matrix keyboards, switches, and LED lights. When the device is set to the training mode, user can control the device through the keyboards to make it generate several types of pulse, and when device is set to the test mode, user 
can choose the answer with the keyboards. The different displays of two LED lights mean different working states of the device.

\section{Artificial hand model}

The hand model is made by the material of TPE. Sensory experiments show that it is more lifelike than the traditional latex[1] or silicone material. Furthermore, the hand feeling is more fine and smooth with a little glutinousness, so it is similar with human hand skin. Inside the hand model, there are hard plastic panels, plastic bag wire ropes, small size latex tubes respectively as a simulation of human body skeleton, blood vessels, arm tendons. The tube wall of latex tube is thin, which can better shows the change of pressure of the internal liquid. The artificial hand is shown in Figure 4.

\section{The design of the software}

The function module diagram of the system is shown in Figure 5.

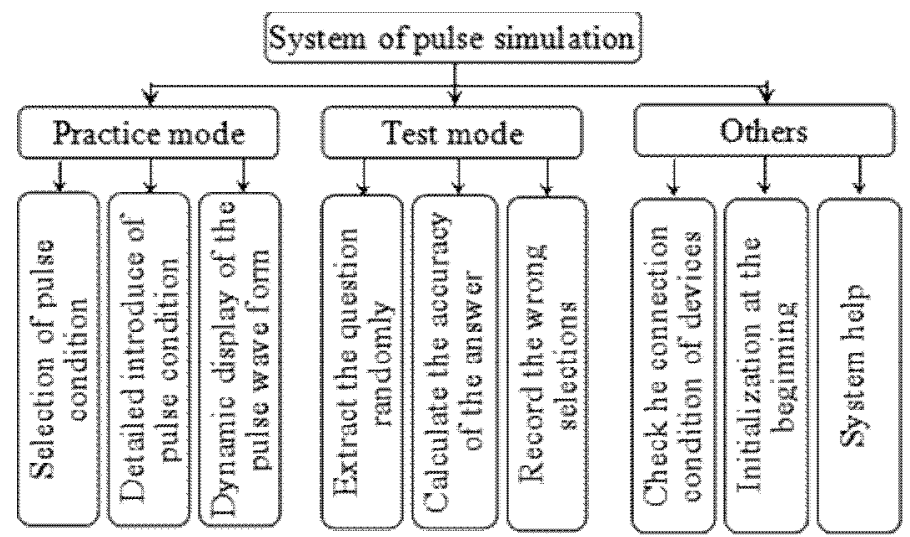

Figure 5 . The system function module diagram

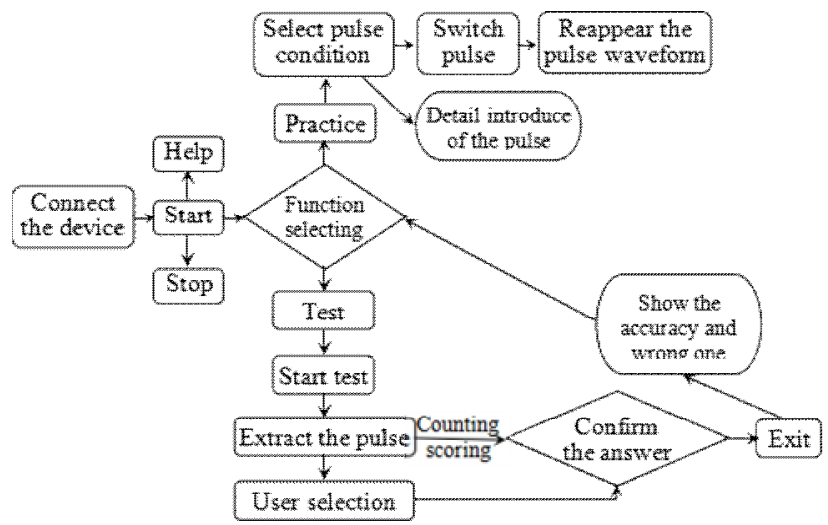

Figure 6. Upper-computer software flow diagram

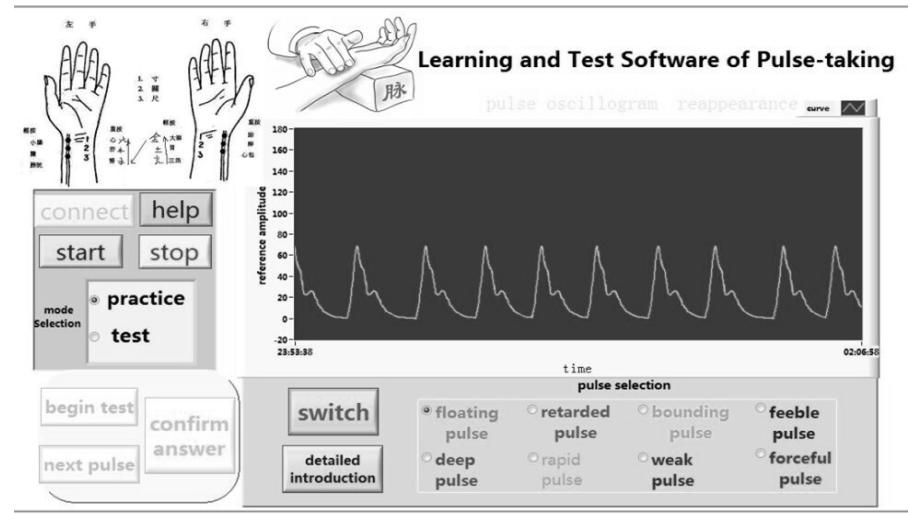

Figure 7. visible control software interface 


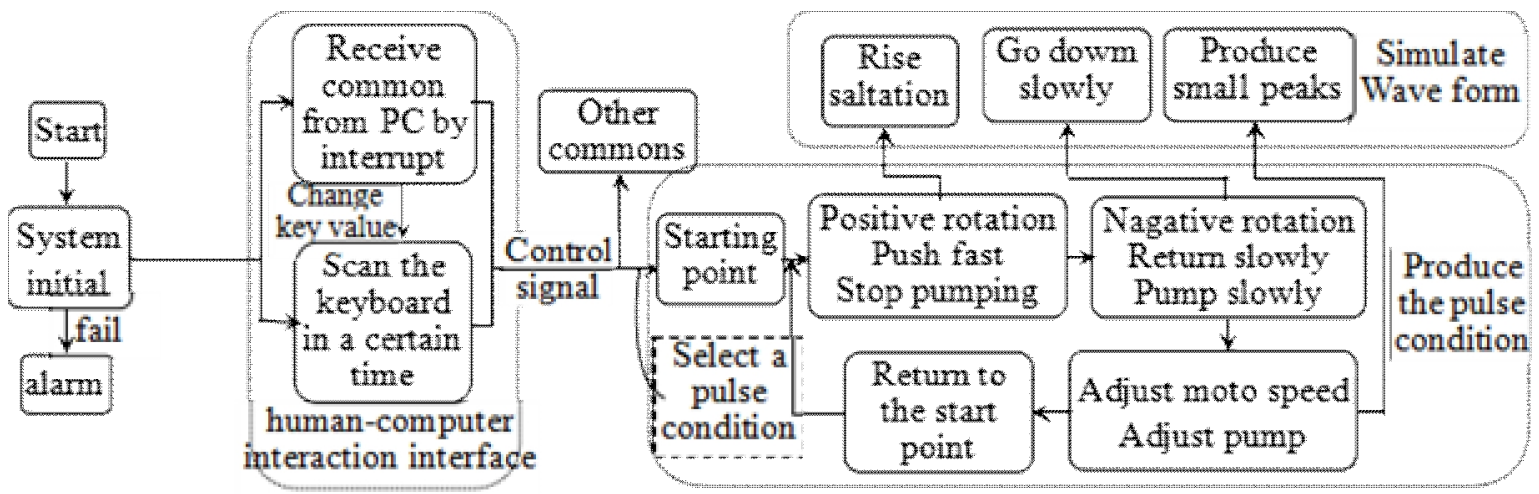

Figure 8. Flow chart of Lower machine

Lower machine control is realized by using STM32 programming[10], while upper-computer software using LabVIEW to develop visible software, and they communicate through the serial port. Upper-computer software includes the functions of pulse-taking practice and test. In the practice mode, users can learn specific human pulse waveform through the equipment by using the software. The software can synchronously display oscillograph on the screen and there is a part for learners to test and evaluate. The flow diagram of upper-computer software is shown in Figure 6 above and the visible control software interface is shown in Figure 7 above.

The main functions of a machine are to control DC motor reversing and rotate speed, pump working frequency, magnetic valve, etc, receiving the signals of man-machine interface and PC hardware, and reading the sensor data to realize the feedback control. Lower machine control program flow chart is shown in Figure 8.

In order to reproduce the human pulse, from the very beginning, the amplitude, frequency, pulse condition rise time, the time that generate lower peak of pulse wave and amplitude from the human pulse wave are extracted and then translated them into control parameters as the system control input parameters. Then the MCU control DC motor rotate quickly based on the technology of PWM speed regulation[11]. Within the given time, motor drives screw-sliding table fast to squeeze the simulation chamber in a specific distance, and the simulation blood vessel would generate a type of mutationally rising pulse condition. During this process, the pump does not work. And then slow down the motor speed to drive sliding table returned slowly, while the pump draw the liquid in the storage tank back to the blood chamber slowly. When the second or third small wave peak arrived, program control motor and the pump speed change together to drop the pressure inside the simulation blood vessel. Finally, during a given period of time, sliding table will return to the starting point, and then adjust control parameters according to the pressure feedback signal and proceed into the next cycle. In motor speed control, of course, to effectively avoid external factors (such as friction between the screw and sliding table), PID control algorithm is adopted.

\section{Results}

Using pulse sensor to test simulation pulse signal, compared with human pulse and the record in TCM, through a large number of experiments, the result shows that the simulation is almost identical to human pulse at high stability. Just as Figure 9 shows. 


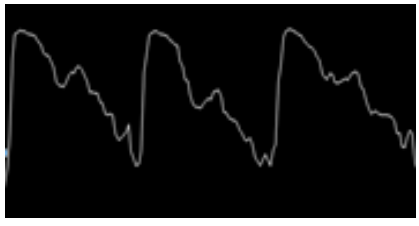

a

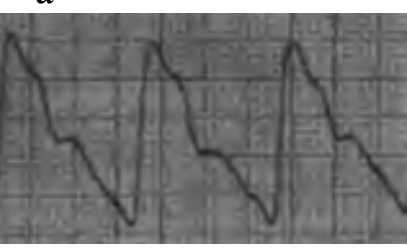

C

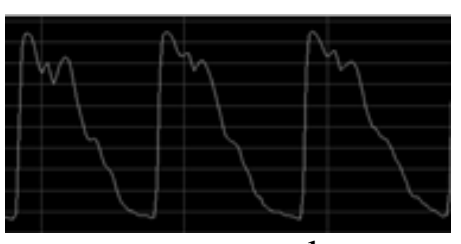

b

Figure 9. pulse waves. a: the pulse-wave detected from the imitate hand; b: the pulse-wave detected from the real hand; c: the pulse-wave recorded in data[12]

This new kind of pulse simulation device realize the imitation of 8 kinds of typical pulse. Since the control parameter of the device can be fine tuning, the device can expend the quantity of imitate pulse. The pulse simulation was detected by famous veteran doctor of TCM with blind detection method. It turns out that the feeling of finger are identify to the real one actually. The simulation results are shown in figure 10, taking the floating-pulse, deep-pulse, retarded-pulse, and rapid-pulse as examples.
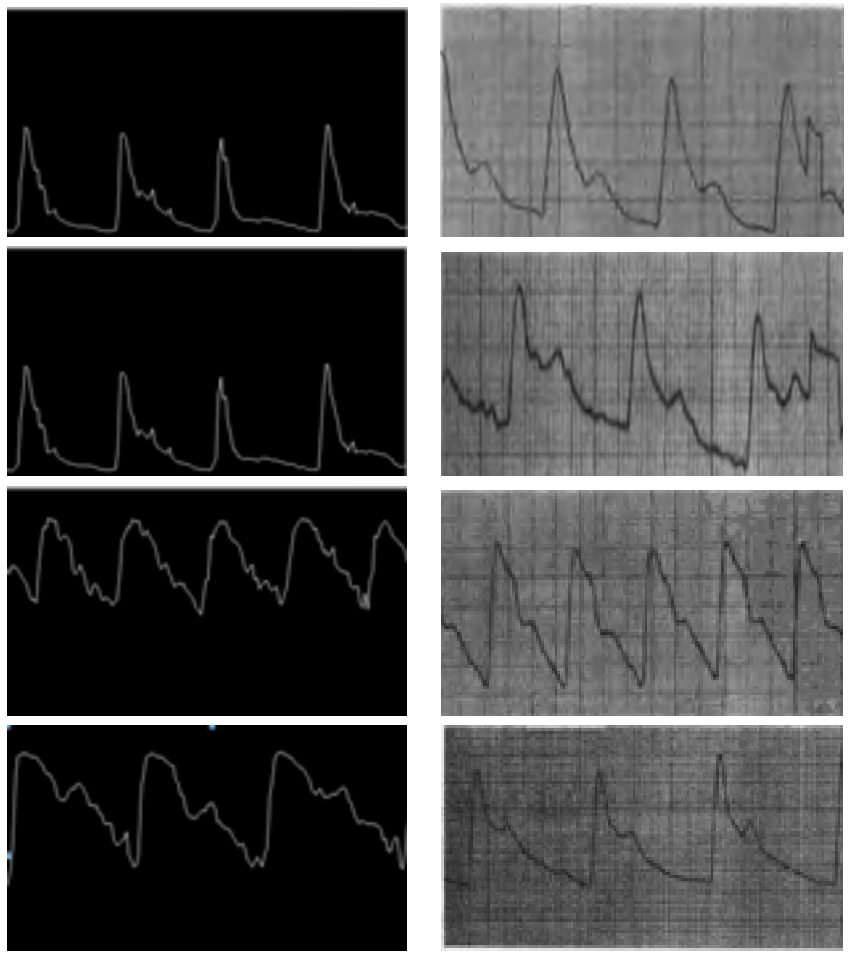

Figure 10. pulse waves.

Notes: In Figure 10, from top to bottom are floating-pulse, deep-pulse, retarded-pulse, and rapidpulse. And the imitate pulse in simulation hand are shown on the left, the recorded pulse in database are shown on the right.

\section{Summary}

This device emphasizes on the high-quality but low-cost idea to realize the simulation of human pulse condition, By improving the control algorithm, it breaks down the barrier that traditional pulse simulation device must adopt precise but unusual hardware structure and reduces the cost. It can change the pulse potential, frequency, pulse shape and so on to realize multiple pulse condition in 
high precision by adjusting the parameters. Visualization software interface is intuitive and can see a detailed introduction of the pulse condition and the characteristics of pulse condition waveform clearly, which can improve the quality of TCM education and efficiency of pulse-taking learning. The simulation wave is nearly agreed with the human pulse. Additionally, the device provides a user-friendly interface. Also, this device is easy to operate, move and maintain because of its simple mechanical structure. It will become an auxiliary tool of TCM education and learning. This device solves the problem that pulse instrument is not easy to widespread use. Hopefully, this study will help to roll out pulse simulation device in teaching, learning and be a catalyst for development of traditional Chinese medicine.

\section{References}

[1]Tang, W.CH. \& Sun, H.J. \& Li, B.F. 2000. The study on traditional Chinese medicine pulse condition simulation system. Chinese Journal of Basic Medicine in Traditional Chinese Medicine 07(2): 105-106.

[2]Li, L. 2002. Pulse simulation device for traditional Chinese medicine education, Chinese patent CN1115054.8.

[3]Shi, Q, et al. 2006. Study on basic pulse of traditional Chinese medicine. The Conference on the Establishment of the Diagnostics Branch of China Association of Chinese Medicine. 228-342.

[4]Heo, Hyun. et al. 2008. Radial Artery Pulse Wave Simulator using a Linear Motor. Engineering in Medicine and Biology Society, 2008. EMBS 2008. 30th Annual International Conference of the IEEE. 4895 - 4898.

[5]Xiao, X.Q. et al. 2011. The design of new pulse simulation system of traditional Chinese medicine. Liaoning Journal of Traditional Chinese Medicine,. 38(9): 1726-1728.

[6]Zhang, F. 2013. Research and design of pulse simulation and reappear. Shanghai: Shanghai Jiao Tong University.

[7]Tang, L.H.(ed.) 2013. Basics of Mechanical Design. Beijing: Electronic Industry Press.

[8]Hou, Q.F. \& Luo, Haibo, Wang, H.F. 2007. The design of large current DC motor drivers based on VNH3SP30. Microcomputer Information. 28:92-94.

[9]Lei, H.M. \& Cheng, Y.Y. 2012. The optimization design of DC moto drivers based on L298N. Digital Technology \& Application. 02: 118+120.

[10]Lu, Y.L.(ed.) 2013. The theory and the design of embedded system based on STM32. Beijing: China Machine Press.

[11]Yang, X.Y.(ed.) 1995. The theory and design of motor speed adjustment(version two). Beijing: China Electric Power Press.

[12]Fei, Y.F.(ed.) 2003. The modern traditional Chinese medicine pulse-taking. Beijing: People's Medical Publishing House. 